\title{
Valorisation des huiles végétales d'origine béninoise par alcoolyse enzymatique
}

Mohamed M. SOUMANOU'

Fidèle P. TCHOBO ${ }^{1}$

Aléodjrodo P. EDORH ${ }^{2}$

Georges ACCROMBESSI ${ }^{3}$

${ }^{1}$ Ecole polytechnique d'Abomey-Calavi, Laboratoire de recherche en chimie et biologie appliquées (LARECBA), Unité de recherche en génie enzymatique et alimentaire, Université d'Abomey-Calavi, 01 BP 2009 Cotonou, Bénin <msoumanoufr@yahoo.fr>

2 Faculté des sciences et techniques, Département de biochimie et de biologie cellulaire,

Université d'Abomey-Calavi, 01 BP 526

Cotonou, Bénin

${ }^{3}$ Faculté des sciences et techniques, Laboratoire de chimie organique, Université d'Abomey-Calavi, 01 BP 526 Cotonou, Bénin

Article reçu le 10 avril 2004 accepté le 19 mai 2005

\begin{abstract}
Fatty acid esters synthesized from vegetable oils by alcoholysis reaction are potentially applied in cosmetic and as biodiesel fuel. The alcoholysis of cottonseed oil by lipases from various microorganisms was investigated. Among several microbial lipases tested in this work, some strains showed high hydrolytic activity, allowing activity for alcoholysis reaction. To select suitable alcohols and biocatalysts, alcoholysis screening in $n$-hexane and in a solvent free medium was developed. In organic solvent, only crude lipases from Pseudomonas strain were active in all alcohol tested. The best conversion (93\%) was found with Pseudomonas fluorescens lipase (AK). In a solvent free medium, except Pseudomonas strain lipase, all crude microbial lipases were almost inactivated. To solve such lipase inhibition, a three step protocol addition of methanol was used. By using this enzymatic process, Pseudomonas fluorescens lipase immobilized on polypropylene support displayed high conversion (> 61\%) in various beninese vegetable oils. Thus, giving added value to cheaper vegetable oils.
\end{abstract}

Key words: alcoholysis reaction, lipases, immobilization, natural vegetable oils, organic solvent, fatty acid esters

\section{Introduction}

Les huiles et graisses végétales jouent un rôle important dans l'alimentation grâce à l'apport énergétique qu'elles fournissent et à leur richesse en acides gras essentiels et en vitamines. Outre ce rôle d'agent nourricier, certaines huiles et graisses végétales possèdent des applications thérapeutiques en raison de leurs propriétés biologiques intéressantes [1, 2].

De nos jours, les huiles végétales constituent de précieuses matières premières pour l'industrie. Comme domaines d'application, on peut citer les industries pharmaceutiques, cosmétiques, des détergents, des lubrifiants et des agents tensioactifs (biosurfactants) [3, 4]. L'intérêt accordé à ces produits dérivés réside dans leur taux de biodégradabilité extrêmement élevé [5]. En raison de l'énergie dégagée lors de la combustion, des huiles végétales ont été expérimentées comme biocarburants. Dès 1897, Diesel utilisa l'huile d'arachide pour la mise en marche des engins à moteur diesel [6]. À cause de leurs viscosités très élevées, les huiles végétales ont été abandonnées au profit des monoalkyls d'esters d'huiles végétales. Les esters d'huiles végétales de préférence méthylés et éthylés, sont appelés biocarburants et possèdent des propriétés chimiques très intéressantes.

Ces molécules peuvent être obtenues soit par voie chimique classique soit par voie enzymatique. Les produits synthétisés dans le premier cas ne sont pas souvent purs et nécessitent des opérations supplémentaires de distillation aptes à éliminer les produits secondaires. La plus attrayante méthode correspond à la voie enzymatique, en raison des spécificités que possèdent les biocatalyseurs, réduisant de manière significative la présence de produits secondaires (figure 1) et surtout avec les avantages de la biocatalyse en milieu organique peu hydraté dit non conventionnel $[7,8]$. Au Bénin, malgré la grande diversité des huiles végétales provenant des technologies traditionnelles, artisanales et industrielles, force est de remarquer que très peu de travaux portant sur leur bioconversion en produit à haute valeur ajoutée ont été réalisés. C'est la raison qui justifie le choix du présent travail. L'intérêt porté à cette étude est d'autant plus justifié que les huiles végétales, outre leur utilisation en alimentation et dans la fabrication de savons, sont destinées à l'exportation. Dans ce travail, les aptitudes à l'hydrolyse et à l'alcoolyse de plusieurs lipases microbiennes ont été testées. De plus, la production d'esters d'acides gras à partir des huiles végétales naturelles a été expérimentée. 


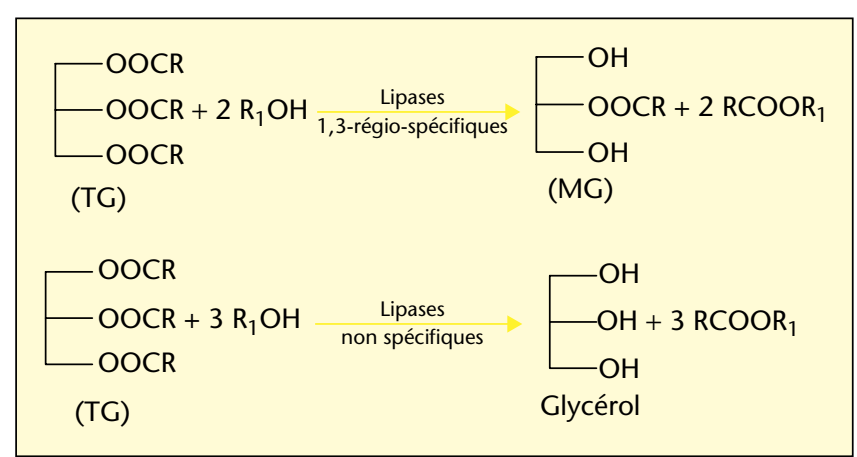

Figure 1. Réactions d'alcoolyse des molécules de triglycérides au moyen des lipases.

\section{Matériel et méthodes}

\section{Matériel biologique}

Le matériel biologique utilisé regroupe sept (7) lipases à l'état brut provenant de Pseudomonas cepacia (PS), Pseudomonas fluorescens (AK), Rhizopus oryzae (RO), Candida rugosa (CR), Penicillium camembertii (PC), Aspergillus niger (AN), Mucor javanicus (MJ) et deux lipases immobilisées commerciales des souches de Rhizomucor miehei (RM) et Thermomyces lanuginosa (TL). Ces différentes lipases microbiennes ont été obtenues par fermentation par les firmes Amano (Japon) et Novonordisk (Danemark). En raison de leurs spécificités très variées, leurs activités hydrolytiques en milieu aqueux ont été déterminées.

\section{Mesure de l'activité hydrolytique des lipases}

Pour la détermination de cette activité, une solution enzymatique est préparée au moyen de $6 \mathrm{mg}$ de lipase dans $2 \mathrm{~mL}$ de solution tampon phosphate à $\mathrm{pH}=7$. La réaction d'hydrolyse est réalisée en utilisant $1 \mathrm{~g}$ d'huile végétale diluée dans $5 \mathrm{~mL}$ d'heptane dans laquelle on ajoute 20 $\mu \mathrm{L}$ de solution de chlorure de calcium $(0,2 \mathrm{M})$ et $50 \mu \mathrm{L}$ de solution enzymatique. Le mélange ainsi obtenu est maintenu à $37^{\circ} \mathrm{C}$ et homogénéisé au moyen d'un agitateur magnétique à une vitesse de rotation de $300 \mathrm{rpm}$ pendant $30 \mathrm{~min}$. La réaction s'arrête lorsqu'on y ajoute $3,5 \mathrm{~mL}$ d'un mélange composé d'acétone/éthanol (1:1, v/v). L'acidité libre est titrée avec une solution décinormale de soude au moyen de la phénolphtaléine comme indicateur coloré. L'activité enzymatique ainsi déterminée exprime le nombre de mole d'acides gras libres que libère $1 \mathrm{mg}$ de lipase par minute.

\section{Immobilisation des lipases}

Les lipases microbiennes testées dans le présent travail ont été immobilisées de manière adsorptive afin d'augmenter leur stabilité, notamment en milieu organique. Avant l'immobilisation proprement dite, un traitement préalable du support est nécessaire. Ainsi, $0,5 \mathrm{~g}$ de support à base de polypropylène est introduit dans $2 \mathrm{~mL}$ d'alcool éthylique pendant quelques minutes. Une quantité de $0,5 \mathrm{~g}$ de lipase dissoute dans $20 \mathrm{~mL}$ de $0,1 \mathrm{M}$ de solution de tampon phosphate $(\mathrm{pH}=7)$ est ajoutée au support prétraité. Le mélange obtenu est agité à température ambiante pendant 12 h à 200 rpm. La lipase immobilisée est collectée par filtration sous vide et lavée deux fois avec $20 \mathrm{~mL}$ de solution tampon puis avec de l'eau distillée $(20 \mathrm{~mL})$. Le produit extrait déshydraté au lyophilisateur pendant $12 \mathrm{~h}$ constitue la lipase immobilisée.

\section{Détermination de l'acidité libre et de la teneur en eau des huiles végétales}

Pour une meilleure appréciation de ces huiles, I'acidité libre exprimée en pourcentage d'acide oléique a été déterminée. La teneur en eau et matières volatiles des huiles a été évaluée par la méthode de chauffage [9].

\section{Alcoolyse enzymatique}

La réaction d'alcoolyse expérimentée dans cette étude a été réalisée au moyen de lipases microbiennes sous des formes libre et immobilisée aussi bien en milieu organique qu'en milieu dépourvu de solvant.

\section{Alcoolyse en milieu organique}

Dans des tubes de $5 \mathrm{~mL}$, on introduit 0,25 mmol d'huile avec 0,75 mmol $\mathrm{d}^{\prime}$ alcool dans $2 \mathrm{~mL}$ de $n$-hexane comme solvant organique. La réaction $s^{\prime}$ effectue en milieu fermé sous agitation magnétique à $300 \mathrm{rpm}$ à $30^{\circ} \mathrm{C}$ pendant 24 heures en présence de différents types de lipases sous des formes libre et immobilisée. Des quantités d'enzyme à $5 \%, 10 \%$ par rapport à la masse d'huile, ont été utilisées respectivement pour les lipases libres et immobilisées. Pour l'étude cinétique, des échantillons du milieu réactionnel $(5 \mu \mathrm{L})$ ont été prélevés à des intervalles de temps donné puis dilués dans $n$-hexane pour l'analyse chromatographique.

\section{Alcoolyse en milieu sans solvant}

La réaction est initiée en ajoutant des quantités de lipases définies plus haut dans un mélange de $0,25 \mathrm{mmol} \mathrm{d}^{\prime}$ huile et $0,75 \mathrm{mmol} \mathrm{d}^{\prime}$ alcool et $s^{\prime}$ effectue sous agitation à $40^{\circ} \mathrm{C}$ à $300 \mathrm{rpm}$. Des échantillons $(25 \mu \mathrm{L})$ ont été prélevés périodiquement du milieu réactionnel puis dissous dans $1 \mathrm{~mL}$ de $n$-hexane. La solution obtenue est filtrée avant l'injection au gaz chromatographe.

\section{Analyse chromatographique}

Les analyses qualitatives et quantitatives ont été réalisées afin d'apprécier la réaction enzymatique.

\section{Analyse qualitative par chromatographie sur couche mince (CCM)}

L'analyse qualitative au moyen de la chromatographie sur couche mince a été effectuée sur des plaques à base de gel de silice de la firme Fluka. Pour la séparation des glycérides et esters d'acides gras, les échantillons d'huile ont été dilués dans l'éther de pétrole. Le mélange composé d'éther de pétrole, d'éther éthylique et d'acide acétique dans les proportions (85:15:1, v/v/v) a été utilisé comme système de solvant d'élution et pour une meilleure séparation des produits, les plaques ont été révélées au moyen d'une solution d'acide sulfurique $50 \%$ puis chauffées à $105^{\circ} \mathrm{C}[10]$.

\section{Analyse quantitative par chromatographie en phase gazeuse $(C P G)$}

La détermination de la composition des acides gras des huiles végétales testées dans le présent travail a été déterminée par CPG basée sur les esters méthyliques obtenus par méthanolyse alcaline. Ainsi, $10 \mathrm{mg}$ d'huile sont dissous dans $1 \mathrm{~mL}$ de $n$-heptane puis méthylés par $20 \mathrm{~mL}$ de la solution de méthylate de sodium $(0,5 \mathrm{M})$. Le mélange obtenu est agité pendant une minute et après centrifugation, $1 \mu \mathrm{L}$ de la phase surnageante est injecté au gaz chromatographe équipé d'une colonne capillaire BP X70 (de 0,25 $\mu \mathrm{m}$ d'épaisseur du film, de $25 \mathrm{~mm}$ de longueur et de $0,25 \mathrm{~mm}$ de diamètre intérieur) et d'un détecteur à ionisation de flamme. L'analyse a été conduite à des températures programmées de $150^{\circ} \mathrm{C}$ à $250^{\circ} \mathrm{C}$ avec un gradient de température de $5^{\circ} \mathrm{C} / \mathrm{min}$. L'injecteur et le détecteur sont maintenus à $250^{\circ} \mathrm{C}$ utilisant l'hydrogène comme gaz vecteur. Les acides gras sont identifiés sur la base de leurs temps de rétention et quantifiés au moyen des standards purs d'esters méthlyliques d'acides gras.

Les esters d'huiles végétales obtenus au cours de l'alcoolyse lipasique aussi bien en milieu organique qu'en absence de solvant ont été carac- 
Tableau 1. Acidité et teneur en eau et matières volatiles de quelques huiles végétales.

\begin{tabular}{|llcc|}
\hline $\begin{array}{l}\text { Huiles } \\
\text { végétales }\end{array}$ & $\begin{array}{c}\text { Méthode } \\
\text { de } \\
\text { production }\end{array}$ & $\begin{array}{c}\text { Acidité } \\
\mathbf{( \% )}^{\mathbf{a}}\end{array}$ & $\begin{array}{c}\text { Teneur en eau et } \\
\text { matières volatiles } \\
\mathbf{( \% )}\end{array}$ \\
\hline $\begin{array}{l}\text { Huile de coton } \\
\text { Huile de palme } \\
\text { raffinée }\end{array}$ & $\begin{array}{l}\text { Industrielle } \\
\text { Industrielle }\end{array}$ & $0,10 \pm 0,01$ & $0,06 \pm 0,02$ \\
$\begin{array}{l}\text { Oléine de palme } \\
\text { Huile d'arachide }\end{array}$ & Industrielle & $0,65 \pm 0,03$ & $0,15 \pm 0,03$ \\
Huile de & Traditionnelle & $2,42 \pm 0,15$ & $0,42 \pm 0,05$ \\
tournesol & Artisanale & $1,15 \pm 0,06$ & $0,43 \pm 0,07$ \\
Huile de coco & Traditionnelle & $1,23 \pm 0,07$ & $0,84 \pm 0,08$ \\
Huile de palmiste & Traditionnelle & $2,3 \pm 0,17$ & $1,52 \pm 0,15$ \\
\hline
\end{tabular}

${ }^{a}$ Acidité libre exprimée en pourcentage d'acide oléique.

térisés après injection $\mathrm{d}^{\prime} 1 \mu \mathrm{L}$ de milieu réactionnel et de pentadécanoate de méthyle comme standard interne au gaz chromatographe. La synthèse est suivie au cours de l'incubation par la détermination du taux de conversion en ester à partir de la quantité d'esters d'acides gras formés.

\section{Résultats et discussion}

\section{Étude de la qualité des huiles végétales testées}

Cette étude repose sur la détermination de l'acidité libre d'une part et de la mesure de la teneur en eau et matières volatiles des huiles végétales d'autre part. Les valeurs obtenues pour ces deux paramètres sont présentées dans le tableau 1.

La détermination de l'indice d'acide permet d'apprécier le degré d'altération par hydrolyse des huiles. Les valeurs de l'acidité libre sont comprises entre 0,10 pour l'huile de coton la moins hydrolysée et 2,3 pour I'huile de palmiste la plus altérée. L'acidification des huiles végétales après leur production peut se produire par hydrolyse chimique ou enzymatique lorsque le corps gras se trouve en présence d'eau dans les conditions favorables [11]. Les valeurs obtenues sur les échantillons d'huiles testées sont assez faibles car représentent moins de $3 \%$. De plus, on remarque que les huiles les moins acides proviennent des unités industrielles de production, en raison des traitements favorisant la séparation de la majeure partie des acides gras libres.

La teneur en eau et en matières volatiles des huiles, facteur important d'hydrolyse a été déterminée. Les taux obtenus $(0,06 \%$ à $1,5 \%)$ sont faibles et confirme la faible acidité des huiles.

En somme, on note une pureté des huiles testées et par conséquent aptes à la réaction d'alcoolyse.

\section{Activités hydrolytiques des lipases microbiennes}

Avant la conduite des réactions d'alcoolyse proprement dite, les pouvoirs hydrolytiques de sept (7) lipases microbiennes vis-à-vis de quatre (4) huiles végétales ont été déterminés et sont illustrés dans la figure 2 . De cette figure, il ressort que la lipase de Pseudomonas cepacia (PS) présente la plus forte activité hydrolytique $[21,1 \mu \mathrm{mol} / \mathrm{mg} / \mathrm{min}]$ avec l'oléine de palme. De plus, on remarque que I'huile de coco est fortement hydrolysée en présence de toutes les lipases. Ainsi, l'activité hydrolytique dépend

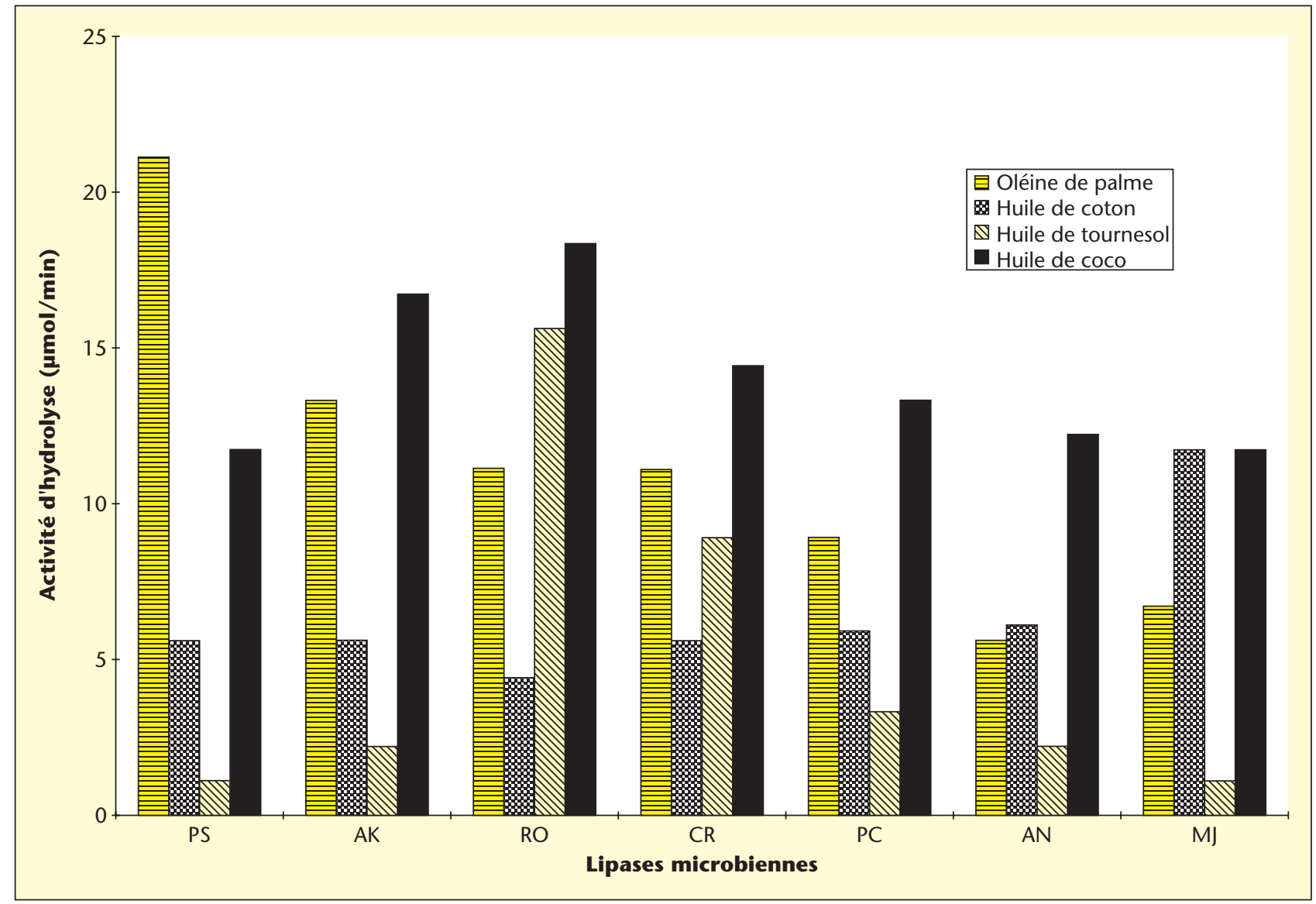

Figure 2. Activités hydrolytiques de lipases microbiennes vis-à-vis de quelques huiles végétales. 
de la nature des acides gras contenus dans les différentes huiles et permet d'expliquer la spécificité des lipases microbiennes. En effet, les meilleures activités hydrolytiques sur les huiles insaturées (huiles de coton et tournesol) ont été obtenues avec Rhizopus oryzae (RO) sur l'huile de tournesol $[15,5 \mathrm{mmol} / \mathrm{mg} / \mathrm{min}]$ et Mucor javanicus (M]) sur l'huile de coton [11,7 $\mu \mathrm{mol} / \mathrm{mg} / \mathrm{min}$ ]. Ces différentes performances indiquent les aptitudes des lipases à cliver les liaisons esters des molécules de triglycérides en milieu aqueux, favorisant ainsi la formation des esters d'acides gras en présence des molécules d'alcool.

\section{Alcoolyse enzymatique des huiles végétales}

Un screening des lipases microbiennes sous formes libres et immobilisées pour les réactions d'alcoolyse a été réalisé. Comme réaction standard, l'alcoolyse de l'huile de coton aussi bien en présence de $n$-hexane qu'en milieu dépourvu de solvant a été expérimentée.

\section{Screening des lipases microbiennes}

L'alcoolyse de l'huile de coton au moyen des lipases microbiennes libres et immobilisées a été étudiée et les produits réactionnels, notamment les esters d'huiles végétales de manière qualitative ont été caractérisés par CCM.

Au regard des résultats obtenus, il ressort que seulement les lipases de Pseudomonas cepacia (PS) et Pseudomonas fluorescens (AK) présentent d'activité dans $n$-hexane en présence des différents types d'alcool. En dehors de ces souches, toutes les autres lipases, en particulier la lipase (MJ) très active sur l'huile de coton en milieux aqueux, sont inactives en présence de méthanol et d'isopropanol.

Plusieurs études faisant ressortir des relations entre les activités hydrolytiques en milieu aqueux et les activités synthétiques en milieu non conventionnel ont été menées [12-15]. Des analyses des résultats de ces travaux, il ressort que les données sur les activités hydrolytiques des enzymes ne permettent pas de prédire le comportement des biocatalyseurs en milieu organique pour leurs activités synthétiques.

En fait, compte tenu de la nature protéique des enzymes microbiennes, beaucoup de lipases testées dans ce travail ont été inactivées en présence de $n$-hexane et d'alcool, à l'exception de la souche Pseudomonas qui présence une forte résistance aux solvants organiques, confirmant ainsi les résultats de Heipieper et al. [16].

En milieu sans solvant organique, on observe après immobilisation une forte apparition des esters d'acides gras par rapport aux résultats de I'alcoolyse en présence de solvant. En effet, l'influence de l'immobilisation sur l'activité enzymatique a fait l'objet d'un certain nombre de travaux, notamment en milieu dépourvu de solvant organique $[11,17]$. À l'usage industriel, cette technique permet non seulement de réduire les coûts de solvant organique, mais aussi de réduire l'inactivation des enzymes et par conséquent d'augmenter leur stabilité. Dans le présent travail, l'alcoolyse semble être plus prononcée avec les lipases immobilisées et ceci est conforme aux résultats de Hsu et al. [18].

Parmi les alcools primaires testés, le méthanol semble inactiver la quasitotalité des lipases testées. Des études antérieures décrites par Mittelbach [19] et Basri et al. [20] ont montré l'effet inhibiteur du méthanol sur certaines souches de lipases, notamment en absence de solvant organique. Néanmoins, les lipases des souches de Pseudomonas résistent aux fortes teneurs en méthanol [21, 22]. Selon certains auteurs, la méthanolyse devient favorable lorsque le méthanol se trouve complètement dissout dans le milieu réactionnel [23, 24].

Après cette étape de screening, les performances cinétiques des lipases immobilisées de Pseudomonas fluorescens (AK) et Pseudomonas cepacia (PS) ont été comparées à celles de deux lipases immobilisées commerciales des souches Rhizomucor miehei (RM) et de Thermomyces lanuginosa (TL) de la firme Novo.

\section{Évolution du taux de conversion}

Une étude cinétique de la méthanolyse en milieu organique de l'huile de coton au moyen de quatre lipases immobilisées présentées plus haut a été effectuée. Les résultats obtenus après analyse par CPG, illustrés par la figure 3, montre que le meilleur rendement (93\%) est atteint avec la lipase AK après 48 heures de réaction. Cependant, au niveau des lipases PS, RM et TL, les taux de conversion sont élevés et atteignent respectivement 77,83 et $87 \%$. En général, au niveau de toutes les lipases testées, les courbes obtenues sont caractérisées par deux phases: une phase à évolution assez rapide du taux de conversion les dix premières heures, puis une autre à évolution très lente. Malgré le rendement très élevé atteint avec la lipase AK, la souche TL semble présenter la meilleure vitesse initiale.

Dans ce milieu réactionnel, l'emploi du solvant organique conduit aux taux de conversion élevés, mais les coûts supplémentaires occasionnés et surtout leur toxicité limitent l'application de ce procédé enzymatique à l'échelle industrielle [24].

En absence de solvant organique, la catalyse enzymatique offre beaucoup d'avantages tels que la réduction en coûts d'extraction de solvant et des possibilités d'utiliser des concentrations élevées en réactifs, augmentant par conséquent la productivité volumique [25]. En utilisant ce procédé, l'influence du rapport molaire (méthanol/huile de coton) sur le taux de conversion a été étudiée. Le tableau 2 présente les résultats obtenus au cours de l'alcoolyse effectuée à $40{ }^{\circ} \mathrm{C}$ pendant $24 \mathrm{~h}$, en faisant varier la quantité de méthanol pour une mole d'huile végétale, au moyen des lipases immobilisées. De cette étude, il apparait que le rapport molaire en substrats influence fortement le rendement et ceci dépend de la souche d'enzyme. En effet, la lipase AK présente une résistante à de

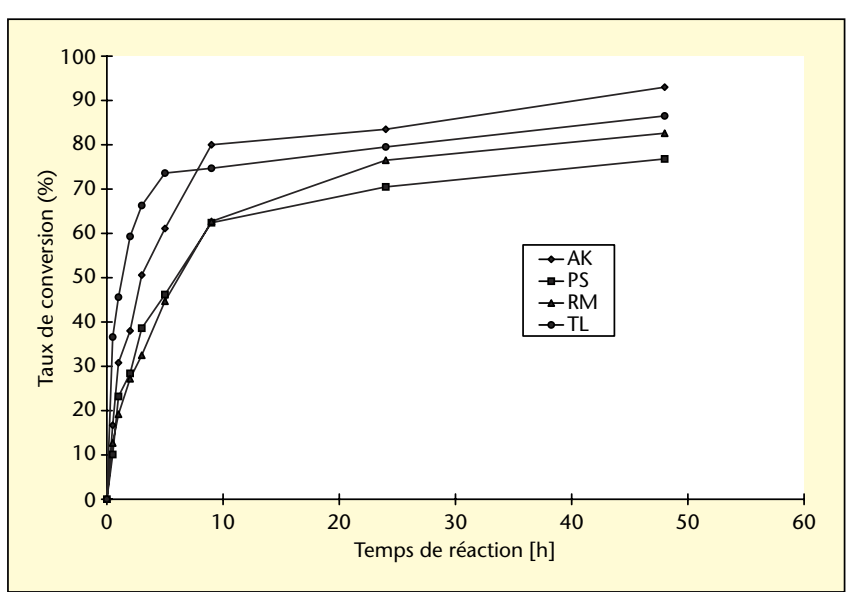

Figure 3. Évolution du taux de conversion au cours de la méthanolyse de I'huile de coton au moyen de lipases microbiennes immobilisées.

Tableau 2. Effet de la teneur en méthanol sur le taux de conversion de l'alcoolyse de I'huile de coton au moyen des lipases immobilisées.

\begin{tabular}{|c|c|c|c|c|}
\hline \multirow{2}{*}{$\begin{array}{l}\text { Rapport molaire } \\
\text { (méthanol/huile) } \\
\text { [mol/mol] }\end{array}$} & \multicolumn{4}{|c|}{$\begin{array}{c}\text { Taux de conversion } \\
(\%)\end{array}$} \\
\hline & AK & PS & RM & TL \\
\hline 3 & 44,2 & 40,2 & 42,0 & 14,6 \\
\hline 3,5 & 50,7 & 45,9 & 5,0 & 10,2 \\
\hline 4 & 82,5 & 76,5 & 2,8 & 2,7 \\
\hline 4,5 & 96,2 & 85,2 & 0,2 & 1,6 \\
\hline 5 & 20,4 & 15,5 & 0,05 & 0,7 \\
\hline
\end{tabular}




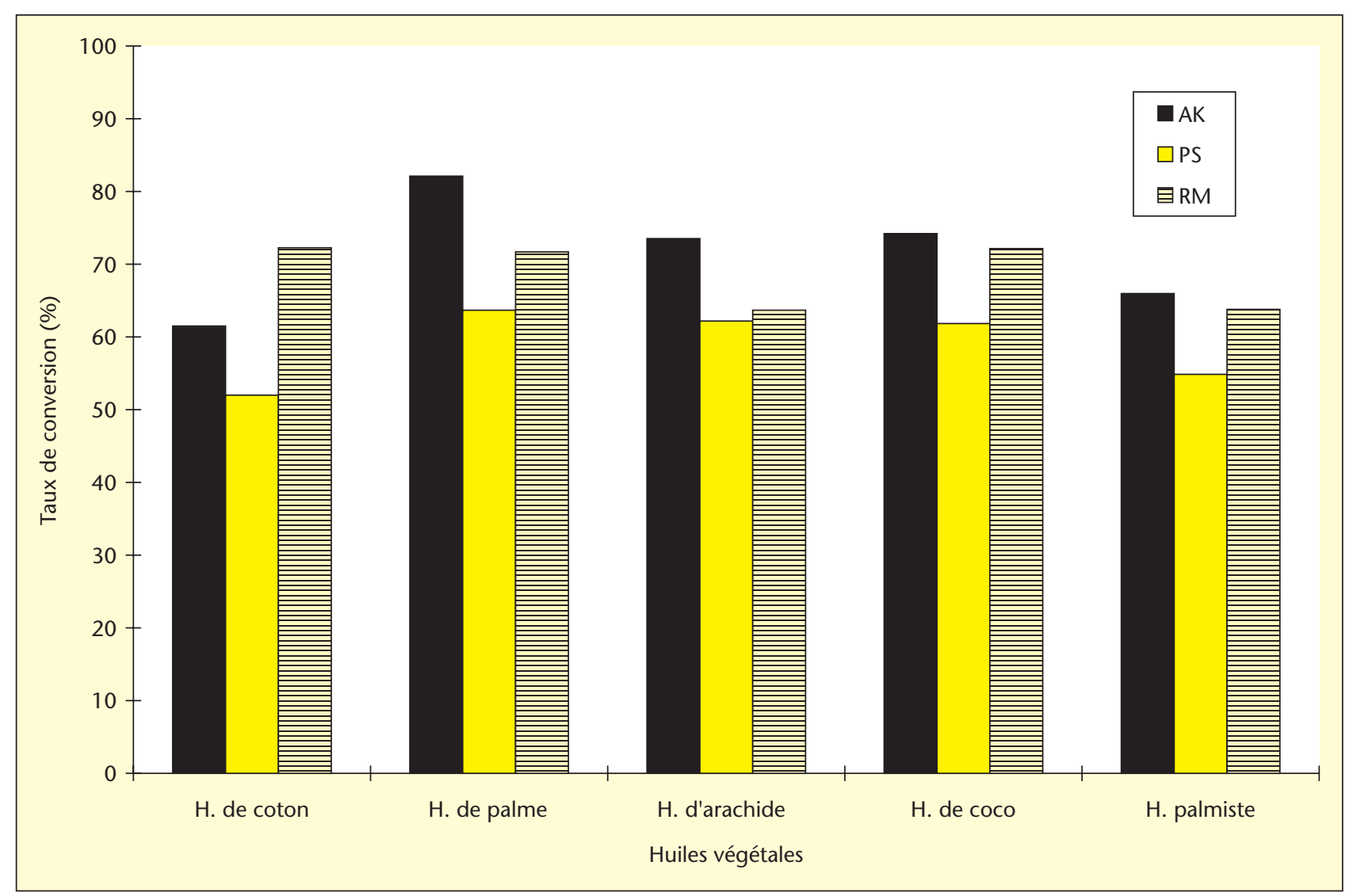

Figure 4. Effet des ajouts successifs de méthanol sur le taux de conversion de l'alcoolyse des huiles végétales au moyen de trois lipases microbiennes immobilisées après $24 \mathrm{~h}$ de réaction.

fortes concentrations de méthanol conduisant ainsi à un rendement de $97 \%$ avec un rapport molaire de 4,5. Au-delà du rapport molaire 3, les lipases RM et TL sont presque inactives. Cette diminution du rendement pourrait être imputée à une dénaturation de ces souches de lipase par modification des liaisons hydrogènes, des interactions électrostatiques et hydrophobes, nécessaires pour le maintien de leur conformation active tridimensionnelle. Ainsi, pour chaque lipase microbienne une certaine quantité d'eau est nécessaire au biocatalyseur pour maintenir cette conformation. En se basant sur l'activité de l'eau du milieu réactionnel, de nombreuses études visant à optimiser le rendement en esters au moyen de lipases immobilisées ont été faites [26-28]. Les taux de conversion optimale obtenus dans la plupart de ces travaux sont atteints lorsque le milieu réactionnel est maintenu à des activités d'eau inférieures à 0,5.

Dans le présent travail, l'optimisation du taux de conversion a été initiée en utilisant un procédé basé sur des ajouts successifs de méthanol au milieu réactionnel décrit par Shimada et al. [22]. Ainsi, la réaction est initiée avec une mole d'huile végétale et une mole de méthanol. Après $4 \mathrm{~h}$ de réaction, on ajoute au milieu réactionnel la seconde mole de méthanol. L'ajout de la troisième mole s'effectue après $8 \mathrm{~h}$ de réaction. Les résultats obtenus avec quelques huiles végétales et présentés dans la figure 4, mettent en évidence l'effet positif des ajouts successifs de méthanol dans le milieu réactionnel. En effet, le taux de conversion est nettement amélioré (> $51 \%$ ) par rapport à la méthode utilisant les trois moles de méthanol en une étape ( $<45 \%$ ) (tableau 2). La lipase AK immobilisée dans le présent travail présente des performances presque similaires pour certaines huiles végétales à celles de la lipase immobilisée commerciale RM.
Devant ce résultat très encourageant, des possibilités de biotransformation des huiles végétales d'origine béninoise en esters méthylés s'ouvrent et permettent de donner une valeur ajoutée à ces matières premières, exclusivement destinées à l'alimentation.

\section{Conclusion}

Au cours de ce travail, I'acidité libre et la teneur en eau et matières volatiles de quelques huiles végétales ont été déterminées. Cette évaluation a permis d'apprécier la qualité de ces huiles avant la mise en œuvre des réactions enzymatiques.

Au regard des résultats obtenus, les huiles végétales testées possèdent des acidités libres et des teneurs en eau faibles.

Les lipases microbiennes testées dans cette étude ont conduit à des résultats qui varient selon la composition du mélange réactionnel. $D$ 'une manière générale, l'immobilisation des lipases sur support à base de polypropylène augmente considérablement l'activité enzymatique en présence de $n$-hexane. En l'absence de solvant organique, l'ajout du méthanol au milieu réactionnel à des intervalles de temps réduits permet d'optimiser le taux de conversion.

Compte tenu de l'intérêt croissant des esters d'huiles végétales dans bien de domaines, l'alcoolyse enzymatique des huiles végétales ne pourrait être qu'une voie de valorisation des huiles végétales naturelles.

Remerciements. Les auteurs remercient le Conseil scientifique de I'Université d'Abomey-Calavi pour le financement d'une partie de ce travail. Les auteurs tiennent également à remercier le Centre béninois de la recherche 
scientifique et technologique (CBRST) pour l'allocation de recherche attribuée à Monsieur Tchobo Fidèle. Nos remerciements vont également aux firmes Amano (Japon) et Novo (Danemark pour le don à titre gracieux des lipases microbiennes brutes et immobilisées.

\section{RÉFÉRENCES}

1. OSBORN HT, AKOH CC. Structured lipids-novel fats with medical, nutraceutical, and food applications. Comprehensive Reviews in Food Science and Food Safety $2002 ; 3: 93-103$

2. AGBO NG, CHATIGRE KO, RONALD ES. Canarium schweinfurthii Engl. : chemical composition of the fruit pulp. J Am Oil Chem Soc $1992 ; 69$ : 317-20.

3. NELSON LA, FOGLIA TA, MARMER WN. Lipase-catalyzed production of biodiesel. J Am Oil Chem Soc 1996 ; 73 : 1191-5.

4. HSU AF, JONES K, FOGLIA TA, MARMER WN. Immobilized lipase-catalyzed production of alkyl esters of restaurant grease as biodiesel. Biotechnol Appl Biochem $2002 ; 36: 181-6$.

5. ABIGOR RD, UADIA PO, FOGLIA TA, et al. Lipase-catalyzed production of biodiesel fuel from some Nigerian lauric oils. Biochem Soc Trans $2000 ; 28$ : 979-81.

6. KNOTHE G. Historical perspectives on vegetable oil-based diesel fuels. Inform $2001 ; 12$ : 1103-7.

7. ZAKS A, KLIBANOV AM. Enzymatic catalysis in nonaqueous solvents. / Bio Chem $1988 ; 263: 3194-31201$.

8. HALLING PJ. Thermodynamic predictions for biocatalysis in nonconventional media : theory, tests, and recommendations for experimental design and analysis. Enzyme Microb Technol $1994 ; 16$ : 178-206.

9. WOLF JP. ANALYSE ET DOSAGE DES LIPIDES. In : Analyse des constituants alimentaires (J.L. Multon eds). Techniques d'analyse et de contrôle dans les Industries Agroalimentaires. $2^{e}$ Edition. Lavoisier-Tec \& Doc 1991 : 157-99.

10. HAAS M], SCOTT KM, MARMER WN, FOGLIA TA. In situ alkaline transesterification : an effective method for the production of fatty acid esters from vegetable oils. J Am Oil Chem Soc $2004 ; 81$ : 83-9.

11. MALCATA FX, REYES HR, GARCIA HS, HILLAND CG, AMUNDSON JH. Immobilized lipase reactions for modification of fats and oils. J Am Oil Chem Soc $1990 ; 67: 890-910$

12. ATOMI H, BORNSCHEUER U. SOUMANOU MM, BEER HD, WOHLFAHRT G, SCHMID RD. Microbial lipases-from screening to design. In : Barnes PJ, ed. Oils-Fats-Lipids, 21st World Congress Int Soc Fat Res England: Bidwater, 1995 : 49-50 ; and Associates, Vol 1.
13. KLIBANOV AM. Why are enzymes less active in organic solvents than in water? Trends Biotechnol 1997; 15 : 97-101.

14. GANDOLFI R, MARINELLI F, LAZZARINI, MOLINARIF. Cell-bound and extracellular carboxylesterase from Streptomyces : hydrolytic and synthetic activities. / Appl Microb $2000 ; 89: 870-5$.

15. MATSUMOTO M, KIDA K, KONDO K. Enhanced activities of lipase pretreated with organic solvents. / Chem Technol Biotechnol $2001 ; 76$ : 1070-3.

16. HEIPIEPER HJ, WEBER F), SIKKEMA I, KEWELOH H, DE BONT JAM. Mechanisms of resistance of whole cells to toxic organic solvents. Trends Biotechnol $1994 ; 12$ : 409-15.

17. CLARK DS. Can immobilization be exploited to modify enzyme activity? Trends Biotechnol $1994 ; 12: 439-43$.

18. HSU AF, JONES K, MARMER WN, FOGLIA TA. Production of alkyl esters from tallow and grease using lipase immobilized in a phyllosilicate sol-gel. J Am Oil Chem Soc $2001 ; 78: 585-8$.

19. MITTELBACH M. Lipase-catalyzed alcoholysis of sunflower oil. / Am Oil Chem Soc $1990 ; 67: 168-70$.

20. BASRI M, HENG AC, RAZAK CNA, ET AL. Alcoholysis of palm oil mid-fraction by lipase from Rhizopus rhizopodiformis. J Am Oil Chem Soc 1997 ; 74 : 113-6.

21. KAIEDA M, SAMUKAWA T, KONDO A, FUKUDA H. Effects of methanol and water contents on production of biodiesel fuel from plant oil catalyzed by various lipases in a solvent-free system. / Biosc Bioeng $2001 ; 91$ : 12-5.

22. SHIMADA Y, WATANABE Y, SAMUKAWA T, ET AL. Conversion of vegetable oil to biodiesel using immobilized Candida antarctica Lipase. I Am Oil Chem Soc $1999 ; 76: 789-93$.

23. SOUMANOU MM, BORNSCHEUER UT. Improvement in lipase-catalyzed synthesis of fatty acid methyl esters from sunflower oil. Enzyme Microb Technol $2003 ; 33: 97-104$.

24. DOSSAT V, COMBES D, MARTY A. Lipase-catalyzed transesterification of high oleic sunflower oil. Enzyme Microb Technol 2002 ; 30 : 90-4.

25. SOUMANOU MM, SCHMID U, BORNSCHEUER UT. Selective production of pure and valuable glyceride using immobilized lipase. I Rech Sci Univ Lomé (Togo) $2002 ; 6: 115-21$

26. UJANG Z, VAIDYA AM. Stepped water activity control for efficient enzymatic interesterification. App Microbiol Biotechnol 1998 ; 50 : 318-22.

27. GRAILLE J. Providing biocatalysis through customizing lipases by different processes. OCL $1999 ; 6: 365-72$ 